\title{
Effects of a 10-weeks strength training program on pain intensity, muscle endurance and kinesophobia in patients with non-specific low back pain
}

\author{
- Elvis I. Agbonlahor ${ }^{1}, \bullet$ Abiola Tolulope Subulade ${ }^{2}$ \\ ${ }^{1}$ Department of Human Kinetics and Sports Science, University of Benin, Benin City, Nigeria. \\ ${ }^{2}$ Department of Physiotherapy, Federal Medical Centre, Owo, Ondo State, Nigeria.
}

\begin{abstract}
The purpose of this study was to establish the effects of a 10-week strength training program on pain intensity reduction, muscle endurance and kinesophobia in patients with non-specific LBP in a tertiary health institution. This study was a pre-test, post-test control group design. A total of 53 patients with LBP participated successfully in the study. The anthropometric parameters were taken and pain intensity was measured using a visual analog scale in which the participants' response to the level of pain was recorded; the fear of movement was measured with Tampa scale kinesiophobia Questionnaire and the muscle endurance with Sorensen back muscle endurance test for both the control and experimental groups before and following a 10-week muscle strength training program. Descriptive statistics of mean and standard deviation were used to summarize the anthropometric profile of the participants while inferential statistics of one-way analysis of variance (ANOVA) was used to test the hypotheses. The outcome of this study indicated that factors such as pain intensity and muscle endurance were found to significantly respond to muscle strength training program, and kinesiophobia did not significantly respond. It was concluded that muscle strength training program can substantially reduce the pain and increase the muscle endurance of patients with LBP. The study, therefore, revealed that the lumbar muscle strength training program is a key element in improving the pain and muscle endurance of patients with LBP.
\end{abstract}

Keywords. Kinesiophobia, muscle endurance, nonspecific low back pain, pain intensity, strength training.

\section{Introduction}

Despite the progress made in the past to avert the occurrence of low back pain (LBP), the incidence is still on the increase in recent times. This may be due to poor awareness of LBP risk factors and poor health care facilities against the backdrop of an unstable/recessed economy, as treatment can linger for years. Yet, it can be significantly reduced, with millions of lives saved and untold suffering avoided, through early detection /reduction in its risk factors and timely intervention. LBP is a very common health problem amongst the population and a major cause of disability that affects work performances and well-being. In the fact, LBP has been regarded as the most common musculoskeletal problem in the world, which affects people across various strata of the society including health care providers in the health institution. Also, LBP is a common symptom of various clinical entities because it can occur alone or in association with other somatic complaints and it can, therefore, be acute, sub-acute or chronic. Nevertheless, several risk factors of LBP such as socioeconomic status, occupational posture, depressive moods, obesity, body height, and age have been identified, thus, causes of the onset of LBP remain obscure and diagnosis difficult to make (Hammed et al., 2017; Hammed \& Agbonlahor, 2016).

Moreover, LBP being the most common 
musculoskeletal problem in the workplace is a major cause of work-related disability, which is associated with major costs in terms of health resource usage, worker disability and absenteeism and it has been established as one of the most common reasons for sick leave in the western world (Cunningham et al., 2008). Equally, LBP is a significant public health issue associated with high costs in care, work absenteeism, activity limitation and frequent use of health services with approximately $10-20 \%$ of patients having pain lasting more than 3 months which is termed Chronic LBP (See, Costa et al., 2013). LBP is a complex disorder, affecting both the physical and psychosocial aspects of the patient. Its management involves the treatment of pain, improving functional capacity and reduction in psychosocial problems such as depression, anxiety, fear avoidance and negative beliefs (Delitto et al., 2012).

Consequently, from the above, kinesiophobia describes the fear of movement and re-injury. It is an irrational and debilitating fear of physical movement and activity resulting from a feeling of vulnerability to painful injury or re-injury. Clinical studies suggest that an excessively negative orientation toward pain (pain catastrophizing) and fear of movement/re-injury (kinesiophobia) are important in the etiology of chronic LBP and associated disability. Hence, they have been reported to be predictors of chronic LBP and disability (Fritz et al., 2007; Picavet et al., 2002). Furthermore, back extensor muscles are postural muscles that aid in maintaining the upright standing posture and controlling lumbar forward bending. Several studies have reported a significant decrease in back extensor muscle endurance in patients with LBP. It is thought that decreased back muscle endurance causes muscular fatigue and overloads soft tissue and passive structures of the lumbar spine, resulting in LBP (Wilder \& Aleksiev, 1996). The authors also identified poor back extensor muscle endurance as an important risk factor for LBP and Mbada et al. (2011) found out that an increase in pain intensity is associated with decrease static back extensors endurance. There is some evidence that decreased muscular endurance could be both a cause and a consequence of LBP. This stresses that weak muscles and/or trunk extensor-to-flexor muscle imbalance are major contributors to the etiology of back pain (Alaranta et al., 2008).

Therefore, testing spinal muscle endurance seems very important in the prediction, prevention, and rehabilitation of LBP. Several types of methods of testing such as static endurance test, active measures of endurance, isokinetic and EMG testing have been studied in the literature (Moreau et al., 2001). From these assessment strategies, isometric endurance testing seems to be cost-effective and requires little equipment in the clinical setting. These features have made investigators focused mainly on isometric endurance assessment. Different static endurance testing methods and evidence regarding their utilization have been reported in the literature. Most common among these methods is prone isometric chest raise test as described by Ito et al. (1996) and prone double straight-leg raise test as described by McIntosh et al. (1998) and prone static chest raise test described by Biering-Sorensen (1984).

Meanwhile, exercise is one of the management strategies that are widely used in LBP in developed nations. It encompasses a heterogeneous group of interventions ranging from general physical fitness or aerobic exercise to muscle strengthening and various types of flexibility or stretching exercises. Strength training, also known as weight training or resistance training has gained popularity in recent years, largely due to its appeal and positive impact on many diverse populations, such as athletic, recreational and clinical communities. Strength training programs are used to achieve many different goals, such as enhancement of athletic performance, reduction of the risk or rehabilitation of injury and improvement of muscular tone, size, strength, and endurance. Therefore, strength training increases the concentration of various hormones and growth-promoting agents in the 
body that may contribute to the improvement of muscular strength and size (American College of Sport Medicine, 2009). Most treatments for LBP have modest efficacy at best (Bogduk, 2004). Exercise is one of the few proven treatments for chronic LBP; however, its effect often varies, and no form is clearly better than another (Van-Tulder et al., 2000). The different treatment modalities engaged in the management of LBP have varying effectiveness. Therefore this study aimed to investigate the effect of 10 -week strength training on pain intensity, muscle endurance and kinesophobia in patients with non-specific LBP in a tertiary health institution.

\section{Methods}

\section{Research Design}

This study is a pre-test and post-test control group experimental design of the effects of a 10-week strength training program on the pain intensity, muscle endurance and kinesiophobia in patients with non-specific LBP.

\section{Sample and Sampling Techniques}

The sample for this study consisted of 54 participants. The convenient sampling technique was used to select the participants for the study. Then, a simple random sampling technique was adopted to randomly assign the participants into 2 groups (Experimental Group and Control Group). The inclusion criteria include the following:(a) Participants diagnosed by the orthopedic surgeons and physician as having a non-specific LBP (b) The low back pain need to have persisted for twelve weeks (c) Participants who could comprehend instructions in English and Yoruba languages (d) Participants with mild to moderate pain or disability, while the exclusion criteria include: (a) Participants with specific spine pathology (such as tuberculosis spine, spinal fracture, and tumor). (b) Patients with co-morbidity may influence overall well-being such as cancer and infections referred pain from internal organs, etc. (c) Pregnant women with LBP. (d) Patients with chronic LBP who are younger than 18 years. This is the age which the individuals are expected to make informed consent

\section{Research Instruments}

The research instruments that were used in this study was an adaptation of Hayes \& Patterson (1921), Miller et al. (2010) and Mclntosh et al. (1998) experimentation protocol.

\section{Method for Data Collection}

Ethical approval of the Health Research Ethics Committee of Federal Medical Centre, Owo was sought and obtained before the commencement of the study. The study was conducted under the Declaration of Helsinki Ethical Principles. The purpose and procedure of the study were explained to the participants and they were informed of their right to withdraw anytime they feel unable to cope with the study. The informed consent form for participants was signed and obtained before the commencement of the study. Then, participants were allocated to 2 groups, experiment and control groups using simple random sampling. The experiment group received lumbar muscle resistance training and the control group received physical treatment inform of Mediwave or infrared therapy with a massage using topical analgesics to the back muscles. Moreover, the data from the study were collected and recorded at baseline and at the end of 10 weeks. The participants' baseline data were recorded on first registration into the study and at the end of 10 weeks of study for both experiment and control groups.

The socio-demographic variables that were obtained in this study are gender, age, occupation, weight, height; the clinical parameters that were measured are their heart rate and blood pressure and the outcome measures of the study to be obtained are pain intensity, kinesiophobia and muscle endurance. The data of the participants were collected by the researcher with the help of 
research assistants following the under listed procedures.

Age: Participants supplied their age as at their last birthday and the obtained value was recorded in years

Height: The participants stood on stadiometer barefooted erect, with back against the bar of stadiometer, knees straight and hands by the sides. Height measurement was taken as the distance between the vertex of the head and the heels and was recorded to the nearest 1.0 centimeter.

Body weight: The body weight of the participant was collected by standing on the weighing scale dressed in light clothes and was recorded in kilograms to the nearest line point.

Blood pressure: The cuff of the sphygmomanometer was wrapped evenly and snugly around the arm of the participants at $2.5 \mathrm{~cm}$ above the site of brachial pulsation. The pressure at which the first sound (korotkoff) is heard will be recorded as systolic blood pressure. The researcher continued with the deflation of the cuff noting the point when the last sound is heard which will be recorded as diastolic blood pressure in $\mathrm{mmHg}$. The researcher finally deflated the cuff and removed it from the participant's arm.

Visual analog scale: The researcher explained the scale to the participant, who was asked to identify his or her present level of pain and the participant's response was recorded. Mark 0 stands for no pain while 10 stands for the most severe pain (Power et al., 1997).

Fear of movement: Tampa Scale Kinesiophobia Questionnaire was used in measuring fear of movement in person with musculoskeletal pain. The TSK consists of 17 statements capturing the idea that the pain is a signal for re-injury because of physical inactivity or certain movements. Participants were asked to fill the questionnaire and indicated their level of agreement on a 4 -point rating scale. The possible range values are 4 (low fear of movement) to 68 (high fear of movement)
Sorensen back muscle endurance test: This procedure was measured in seconds. The participant lied prone on a coach with the waist at the edge and the calf muscle striped to the coach. The back was kept straight and the stop watch was used to measure how long the participants could hold the back in that position. The test was stopped immediately the researcher notice that the back was not able to withstand the pain any longer or the participants complained of inability to endure the position again.

Strengthening exercise using resistance training program: The training combined the use of free weights and body weight for the strengthening of the muscles of the spine (erector spinea) and other associated muscles including abdomen (rectus abdominis, oblique abdominis, transverse abdominis), the hip extensors (gluteal maximus) and flexors (ilio-psoas), and the quadriceps femoris.

\section{Data Analysis}

Descriptive statistics of mean and standard deviation were used to analyze the anthropometric profile of the participants while an inferential statistic of one-way analysis of variance (ANOVA) was used to test the hypotheses, however where there was significance difference Tukey HSD Posthoc test was used to identify the source of significance, and the significance level was set at 0.05. All data were analyzed by using SPSS 20.0 statistic packaged software.

\section{Results}

There is no significant difference in the pain intensity reduction of the participants before and following the 10-week of the strength training program.

Table 1 shows the reduction in the means of pain intensity reduction as described by the participants in both groups following the 10 weeks of exposure to the different treatment modalities with the experiment group mean and standard deviation (M 
$\pm \mathrm{SD}$ ) from $0.607 \pm 0.08$ to $0.39 \pm 0.15$ and control group mean and standard deviation $(\mathrm{M} \pm \mathrm{SD})$ from $0.731 \pm 0.13$ to $0.362 \pm 0.14$, and there is a similarity in the pattern of pain reduction by the groups.

From Table 2, the difference in the pain intensity before and following a 10-week intervention using the physical treatment in the control group and muscle training program in the experiment group was determined using ANOVA. There was a significant between groups $\left(\mathrm{F}_{(3,50)}=24.617 ; \mathrm{p}<0.05\right)$. It, therefore, implies that strength training had a substantial effect on the pain intensity reduction of low back pain patients.

\section{Table 1}

The pain intensity using Visual Analogue Scale (VAS) among the groups at week 0 and 10.

\begin{tabular}{|c|c|c|c|c|c|c|}
\hline \multirow{2}{*}{ Variable } & \multirow{2}{*}{ Groups } & \multirow{2}{*}{ Treatment Period } & \multirow{2}{*}{ Mean } & \multirow{2}{*}{$\mathrm{SD}$} & \multicolumn{2}{|c|}{$95 \%$ Confidence Interval } \\
\hline & & & & & Lower & Upper \\
\hline \multirow[t]{4}{*}{ Pain Intensity } & Experiment & 0 week & 0.607 & 0.08 & 0.56 & 0.66 \\
\hline & & 10 week & 0.393 & 0.15 & 0.31 & 0.58 \\
\hline & Control & 0 week & 0.731 & 0.13 & 0.65 & 0.81 \\
\hline & & 10 week & 0.362 & 0.14 & 0.27 & 0.45 \\
\hline
\end{tabular}

Table 2

Comparison of the pain intensity between groups at week 0 and 10 .

\begin{tabular}{lccccc}
\hline & Sum of Squares & $\mathrm{df}$ & Mean Square & $\mathrm{F}$ & $\mathrm{p}$ \\
\hline Between Groups & 1.236 & 3 & 0.412 & 24.617 & $<0.05$ \\
Within Groups & 0.837 & 50 & 0.017 & & \\
Total & 2.073 & 53 & & & \\
\hline
\end{tabular}

Table 3

The pairwise comparisons of the pain intensity of the participants.

\begin{tabular}{llccc}
\hline (I) Groups & (J) Groups & Mean Difference (I-J) & Std. Error & $\mathrm{p}$ \\
\hline \multirow{4}{*}{ Pre Exercise } & Post Exercise & $.2143\left(^{*}\right)$ & 0.0489 & 0.000 \\
& Pre Control & -0.1236 & 0.0498 & 0.075 \\
& Post control & $.2456\left(^{*}\right)$ & 0.0498 & 0.000 \\
& Pre Exercise & $-.2143\left(^{*}\right)$ & 0.0489 & 0.000 \\
Post Exercise & Pre Control & $-.3379\left(^{*}\right)$ & 0.0498 & 0.000 \\
& Post Control & 0.0313 & 0.0498 & 0.922 \\
& Pre Exercise & 0.1236 & 0.0498 & 0.075 \\
& Post Exercise & $.3379\left(^{*}\right)$ & 0.0498 & 0.000 \\
& Post Control & $.3692\left(^{*}\right)$ & 0.0507 & 0.000 \\
& Pre Exercise & $-.2456\left(^{*}\right)$ & 0.0498 & 0.000 \\
& Post Exercise & -0.0313 & 0.0498 & 0.922 \\
& Pre Control & $-.3692\left(^{*}\right)$ & 0.0507 & 0.000 \\
\hline
\end{tabular}

*. The mean difference is significant at the .05 level. 
Table 4

Descriptive statistics of the means of muscle endurance among the groups at week 0 and 10 .

\begin{tabular}{|c|c|c|c|c|c|c|}
\hline \multirow{2}{*}{ Variable } & \multirow{2}{*}{ Groups } & \multirow{2}{*}{ Treatment Period } & \multirow{2}{*}{ Mean } & \multirow{2}{*}{ SD } & \multicolumn{2}{|c|}{ 95\% C.I } \\
\hline & & & & & Lower & Upper \\
\hline \multirow[t]{4}{*}{ Muscle Endurance } & Experiment & 0 week & 72.14 & 25.43 & 57.46 & 88.82 \\
\hline & & 10 week & 88.5 & 18.53 & 77.8 & 99.2 \\
\hline & Control & 0 week & 44.69 & 28.15 & 27.68 & 61.71 \\
\hline & & 10 week & 75.69 & 21.5 & 62.7 & 88.69 \\
\hline
\end{tabular}

Table 5

The comparison of the pain intensity of in the experiment and the control groups at week 0 and 10 .

\begin{tabular}{lccccc}
\hline & Sum of Squares & df & Mean Square & F & p \\
\hline Between Groups & 13581.84 & 3 & 4527.28 & 8.104 & \\
Within Groups & 27930.75 & 50 & 558.62 & & \\
Total & 41512.59 & 53 & & & \\
\hline
\end{tabular}

Turkey's honesty significant difference Post-hoc test was carried out to determine the difference in variation in the pain intensity of the participants. However, all the pairwise of the mean difference were found to be statistically significant $(p<0.05)$ except pre-control versus pre-exercise group (0.124) and post-control versus post-exercise group $(-0.31)$ as shown in Table 3. This implies that the entire pairwise mean had variation. Therefore, strength training influenced the variation of the pain intensity of the participants.

There is no significant difference in the muscle endurance of the participants before and following 10-weeks of strength training

Table 4 shows the increase in the means of muscle endurance in both groups; the experiment group means and standard deviation $(\mathrm{M} \pm \mathrm{SD})$ from $72.14 \pm 25.43$ to $88.5 \pm 18.53$ and control group mean and standard deviation $(\mathrm{M} \pm \mathrm{SD})$ from 44.69 \pm 28.15 to $75.69 \pm 21.5$. However, the means in the control group is more markedly increased compared to the experiment group, even though the experiment group still has higher means.

The one-way ANOVA was used to determine the significance of the difference in the muscle endurance, before and following a 10-week intervention using the physical treatment in the control group and muscle training program in the experiment group is presented in Table 5. It was observed to be statistically significant $\left(\mathrm{F}_{(3,50)}=8.104\right.$; $\mathrm{p}<0.05)$. This implies that the strength training program had a substantial effect on the muscle endurance of the participants.

When compared the muscle endurance of the participants, all the pairwise of the mean difference were found to be statistically significant $(p<0.05)$ except post-control versus pre-exercise group (3.55), post-control versus post-exercise group (12.81) and pre-exercise versus post-exercise group $(-16.36)$ as shown in Table 6. This implies that the pairwise mean had variation. Therefore, the strength training influenced the variation of the pain intensity of the participants.

There is no significant difference in the participants' kinesiophobia before and following 10 -weeks of the strength training program.

Table 7 shows the decrease in the kinesiophobia mean value in both groups; the experiment group mean and standard deviation $(\mathrm{M} \pm \mathrm{SD})$ from 50.93 \pm 7.731 to $49.5 \pm 7.57$ and control group mean and 
standard deviation $(\mathrm{M} \pm \mathrm{SD})$ from $51.0 \pm 3.63$ to $46.92 \pm 2.9$, though it is more in the control while just marginal in the experiment group.

Table 8 presents the comparison of kinesiophobia among the groups at week 0 and 10 . It was observed to be statistically insignificant
$\left(\mathrm{F}_{(3,50)}=1.342 ; \mathrm{p}>0.05\right)$ Thus, the hypothesis which states that there is no significant difference in the participants' kinesiophobia before and following 10 -weeks of strength training was accepted. This implies that the muscle strength training program did not affect the kinesiophobia of the participants.

Table 6

Pairwise comparisons of muscle endurance in the participants.

\begin{tabular}{llccc}
\hline (I) Groups & (J) Groups & Mean Difference (I-J) & Std. Error & $\mathrm{p}$ \\
\hline Pre Exercise & Post Exercise & -16.357 & 8.933 & 0.271 \\
& Pre Control & $27.451\left(^{*}\right)$ & 9.103 & 0.020 \\
& Post control & -3.549 & 9.103 & 0.980 \\
Post Exercise & Pre Exercise & 16.357 & 8.933 & 0.271 \\
& Pre Control & $43.808\left(^{*}\right)$ & 9.103 & 0.000 \\
& Post Control & 12.808 & 9.103 & 0.501 \\
Pre Control & Pre Exercise & $-27.451\left(^{*}\right)$ & 9.103 & 0.020 \\
& Post Exercise & $-43.808\left(^{*}\right)$ & 9.103 & 0.000 \\
& Post Control & $-31.000\left(^{*}\right)$ & 9.270 & 0.008 \\
Post Control & Pre Exercise & 3.549 & 9.103 & 0.980 \\
& Post Exercise & -12.808 & 9.103 & 0.501 \\
& Pre Control & $31.000\left(^{*}\right)$ & 9.270 & 0.008 \\
\hline
\end{tabular}

*. The mean difference is significant at the .05 level.

\section{Table 7}

Descriptive statistics of the means of kinesiophobia among the groups at week 0 and 10 .

\begin{tabular}{lllcccc}
\hline \multirow{2}{*}{ Variable } & \multirow{2}{*}{ Groups } & \multirow{2}{*}{ Treatment period } & Mean & SD & \multicolumn{2}{c}{$95 \%$ C.I } \\
\cline { 6 - 7 } & & & & & Lower & Upper \\
\hline \multirow{2}{*}{ Kinesiophobia } & Experiment & 0 week & 50.93 & 7.73 & 46.46 & 55.39 \\
& & 10 week & 49.5 & 7.57 & 45.13 & 53.87 \\
& \multirow{2}{*}{ Control } & 0 week & 51 & 3.63 & 48.81 & 53.19 \\
& & 10 week & 46.92 & 2.9 & 45.17 & 48.68 \\
\hline
\end{tabular}

Table 8

Comparision of the kinesiophobia among the groups at week 0 and 10.

\begin{tabular}{lccccc}
\hline & Sum of Squares & df & Mean Square & F & p \\
\hline Between Groups & 143.482 & 3 & 47.83 & 1.342 & $<0.05$ \\
Within Groups & 1781.352 & 50 & 35.63 & & \\
Total & 1924.833 & 53 & & & \\
\hline
\end{tabular}




\section{Discussion}

This study was carried out to investigate the effects of a 10-week strength training on pain intensity, kinesiophobia and back muscle endurance among individuals with non-specific LBP.

The age range of the participants was between 22 and 45 and the mean age of all the participants was 38.4. The mean age is a bit lower compared to previous on LBP, which reported higher mean age and also reported that it is more predominant in the older age group (Petersen et al., 2002). This may be because younger and middle-age individuals are now getting more exposed to the risk factors of LBP at earlier age. The frequency and duration of muscle training used in this study were deemed appropriate to produce demonstrable effects based on previous studies of similar or less training duration (Alli, 2013), hence the findings on pain intensity, muscle endurance, and kinesiophobia are comparable. The study showed a reduction in the pain intensity of participants of the experiment and control groups as reported by some other studies (Melzack \& Vetere, 2012). The reduction in pain intensity between the groups was highly significant $(p=0.0001)$ and was as well significant for both groups in the multiple comparisons in the Post-hoc test (Control, $\mathrm{p}=0.0001$, Experiment, $\mathrm{p}=0.001$ ). The findings on the significant reduction in pain intensity following muscle strength training were reported in a study by Maughan \& Lewis (2010), which supported the findings in this study. Other studies that agree with a significant reduction in pain intensity include findings from Frost et al. (1995) study which reported significant pain intensity reduction following 8 sessions of the muscle training program over 4 weeks, and Torstensen et al. (1998) also recorded a significant reduction in pain intensity.

Not all studies reviewed showed a significant reduction in pain intensity. Findings from some studies did not show a significant reduction in pain intensity following the training program (Bernard, 2003). Alli (2013) also reported that his findings on pain intensity reduction after the muscle training program were not significant, which all contradict the findings in this study. This could be due to the methodology used in some of these studies in which both groups were exposed to physical treatment with the experiment group having extra exposure to muscle training. The physical treatment could have been a confounding factor making the groups too similar, hence the nonsignificance. In some other studies, the likely contributing factors to the inconsistent report could also be due to improper interpretation of the results of the training trial, because the treatment comparison in trials in these studies is of unknown efficacy. Bahago (2015) in his study also reported this to be due to insufficient appreciation by the researchers conducting trials and by reviewers summarizing trials of a wide variety of forms training exercise can take, and also the trials do not control the quality of exercise intervention.

Previous studies demonstrated that certain specific muscles of patients with LBP are weaker than those of healthier individuals (Mayer \&Gatchel, 1988), which has made muscle training program very common in studies on LBP (Rainville et al., 2004).

Further findings from this study from the one way ANOVA analysis between the groups showed a highly significant mean difference of muscle endurance tests $(F=8.104, p=0.0001)$. However, the post hoc test showed the significant difference was only in the control group $(\mathrm{p}<0.05)$ while it was not significant in the experiment group $(p=0.271)$.

The findings from this study showed a decrease in the fear of participants have because of pain (kinesiophobia) in the two groups, though the control group demonstrated more reduction in the fear (week $0=51$, week $10=46.92$ ) than the experiment group (week $0=50.93$, week $10=49.50$ ). The finding is however not significant $(\mathrm{F}=1.342$, $\mathrm{p}$ $=0.271)$ and all the previous studies reviewed as well reported non-significant kinesiophobia (Alli, 2013; Picavet et al., 2002). 


\section{Conclusion}

Finally, the results of the study suggest that the effect of 10-week strength training in patients with LBP might increase muscle endurance and reduce pain intensity. The training program may not any effect on the fear of movement in patients with LBP. Therefore the muscle strength training program is a good training modality for reducing pain intensity and muscle endurance of patients with LBP. It could be recommended that the lumbar muscle strength training program should be considered a key element in the management of patients with LBP and included alongside other conventional physical treatment of LBP among patients with LBP for better outcomes of care.

\section{Acknowledgments}

We appreciate the Federal Medical Center, Owo for giving us the platform to conduct the research.

\section{Conflict of interest}

The authors reported no conflicts of interest.

\section{Funding}

The authors declared that they did not receive any financial support for this study.

\section{References}

Added, M.A., Costa, L.O., Fukuda, T.Y., de-Freitas, D.G., Salomão, E.C., Monteiro, R.L. \& Costa L.C. (2013). Efficacy of adding the Kinesio Taping method to guideline-endorsed conventional physiotherapy in patients with chronic nonspecific low back pain: a randomized controlled trial. BMC Musculoskeletal Disorders, 14, 301.

American College of Sports Medicine. (2009). Progression models in resistance training for healthy adults. Med Science Sports Exercise, 41(3), 687-708.

Bogduk, N. (2004). Management of chronic low back pain. Medical Journal of Australia, 180(2), 79-83.

Cunningham, C., Doody, C. \& lake, C. (2008). Managing low back pain: knowledge and attitude of hospital manager. Journal of Occupational Medicine, 258, 282288

Delitto, A., George, S., Dillen, L., Whitman, J. \& Denninger, T. (2012). Clinical practice guidelines linked to the international classification of functioning, disability, and health from the orthopaedic section of the American Physical Therapy Association. Journal of Orthopaedic \& Sports Physical Therapy, 42(4), 1-57.

Fritz, J.M., Cleland, J.A. \& Childs, J.D. (2007). Subgrouping patients with low back pain: evolution of a classification approach to physical therapy. Journal of Orthopaedic and Sports Physical Therapy, 37, 290-302.

Hammed, I.A. \& Agbonlahor, E.I. (2016). Interdependence of marital status and clinical characteristics of morbidity with health-related quality of life among low back pain patients. Journal of Biomedical Human Kinetics, 8, 159-164.

Hammed, I.A., Akindele, M.O. \& Lawal, I.U. (2017). Sociodemographic correlates of health related quality of life in subjects with non-specific low back pain among Nigerians. American Institute of Science (Frontiers in Biomedical Sciences), 2(3), 11-17.

McKenzie, R.A. (1997). Lumbar spine mechanical diagnosis and therapy. New Zeland: Wright and Carman.

Moore, K.L. \& Dalley, A.F. (2006). Clinical oriented anatomy. 5th ed., Limpincot: William and Wilkin Company.

Picavet, H.S.J., Vlaeyen, J.W.S. \& Schouten, J.S.A.G. (2002). Pain catastrophizing and kinesiophobia: Predictors of chronic low back pain. American Journal of Epidemiology, 156(11), 1028-1034.

Van Tulder, M.W., Malmivaara, A., Esmail, R., \& Koes, B.W. (2000). Exercise therapy for low back pain: A systematic review within the framework of the Cochrane Collaboration Back Review Group. Spine, 25, 2784-96. 